\title{
RAMANUJAN'S CONTRIBUTIONS TO EISENSTEIN SERIES, ESPECIALLY IN HIS LOST NOTEBOOK
}

\author{
BRUCE C. BERNDT ${ }^{1}$ AND AE JA YEE ${ }^{2}$
}

\section{INTRODUCTION}

In contemporary notation, the Eisenstein series $G_{2 j}(\tau)$ and $E_{2 j}(\tau)$ of weight $2 j$ on the full modular group $\Gamma(1)$, where $j$ is a positive integer exceeding one, are defined for $\operatorname{Im} \tau>0$ by

$$
G_{2 j}(\tau):=\sum_{\substack{m_{1}, m_{2} \in \mathbf{Z} \\ m_{1}, m_{2} \neq(0,0)}}\left(m_{1} \tau+m_{2}\right)^{-2 j}
$$

and

$$
\begin{aligned}
E_{2 j}(\tau):=\frac{G_{2 j}(\tau)}{2 \zeta(2 j)} & =1-\frac{4 j}{B_{2 j}} \sum_{k=1}^{\infty} \frac{k^{2 j-1} e^{2 \pi i k \tau}}{1-e^{2 \pi i k \tau}} \\
& =1-\frac{4 j}{B_{2 j}} \sum_{r=1}^{\infty} \sigma_{2 j-1}(r) q^{r}, \quad q=e^{2 \pi i \tau},
\end{aligned}
$$

where $B_{n}, n \geq 0$, denotes the $n$th Bernoulli number, and where $\sigma_{\nu}(n)=\sum_{d \mid n} d^{\nu}$. The latter two representations in (1.1) can be established by using the Lipschitz summation formula or Fourier analysis. For these and other basic properties of Eisenstein series, see, for example, R. A. Rankin's text [45, Chap. 6].

In Ramanujan's notation, the three most relevant Eisenstein series are defined for $|q|<1$ by

$$
\begin{aligned}
& P(q):=1-24 \sum_{k=1}^{\infty} \frac{k q^{k}}{1-q^{k}}, \\
& Q(q):=1+240 \sum_{k=1}^{\infty} \frac{k^{3} q^{k}}{1-q^{k}},
\end{aligned}
$$

and

$$
R(q):=1-504 \sum_{k=1}^{\infty} \frac{k^{5} q^{k}}{1-q^{k}} .
$$

\footnotetext{
${ }^{1}$ Research partially supported by grant MDA904-00-1-0015 from the National Security Agency.

${ }^{2}$ Research partially supported by a grant from the Number Theory Foundation.
} 
Thus, for $q=\exp (2 \pi i \tau), E_{4}(\tau)=Q(q)$ and $E_{6}(\tau)=R(q)$, which have weights 4 and 6 , respectively. The function $P(q)$ is not a modular form. However, if $q=\exp (2 \pi i \tau)$, with $\operatorname{Im} \tau=y$, then $P(q)-3 /(\pi y)$ satisfies the functional equation required of a modular form of weight 2 [45, p. 195]. In his notebooks [43], Ramanujan used the notations $L, M$, and $N$ in place of $P, Q$, and $R$, respectively.

Ramanujan made many contributions to the theory and applications of Eisenstein series in his paper [39], [42, pp. 136-162]. Applications were made to the theory of the divisor functions $\sigma_{\nu}$; the partition function $p(n)$, the number of ways the positive integer $n$ can be represented as a sum of positive integers with the order of the summands irrelevant; and $r_{k}(n)$, the number of representations of the positive integer $n$ as a sum of $k$ squares. Since Ramanujan did not consider any further applications of Eisenstein series to $r_{k}(n)$ in his lost notebook, we shall not further discuss his work on sums of squares. However, very exciting research on formulas for $r_{2 k}(n)$ has recently emerged, and Eisenstein series play a prominent role. First, Z.-G. Liu [30] has found a simplified approach to Ramanujan's work in [39]. Secondly, new infinite classes of formulas for $r_{2 k}(n)$ have been discovered by H. H. Chan [20], S. Milne [31], and K. Ono [32].

Among the most useful results on Eisenstein series established by Ramanujan in [39] are his differential equations [39, eqs. (30)], [42, p. 142]

$$
q \frac{d P}{d q}=\frac{P^{2}(q)-Q(q)}{12}, \quad q \frac{d Q}{d q}=\frac{P(q) Q(q)-R(q)}{3}, \quad \text { and } \quad q \frac{d R}{d q}=\frac{P(q) R(q)-Q^{2}(q)}{2} .
$$

For example, the differential equations for $Q$ and $R$ are needed to prove the following beautiful integral formula found on page 51 in Ramanujan's lost notebook [44], [37], [9, Thm. 3.1, pp. 82-83]. If $Q(q)$ and $R(q)$ are defined by (1.3) and (1.4), respectively, then

$$
\int_{e^{-2 \pi}}^{q} \sqrt{Q(t)} \frac{d t}{t}=\log \left(\frac{Q^{3 / 2}(q)-R(q)}{Q^{3 / 2}(q)+R(q)}\right), \quad|q|<1 .
$$

Several further results in this paper depend upon the differential equations (1.5).

Ramanujan recorded numerous results on Eisenstein series in his notebooks as well. Many are dependent on the following basic theorem in the theory of elliptic functions [3, Entry 6, p. 101]. Let, for $0<x<1$,

$$
q:=\exp \left(-\pi \frac{{ }_{2} F_{1}\left(\frac{1}{2}, \frac{1}{2} ; 1 ; 1-x\right)}{{ }_{2} F_{1}\left(\frac{1}{2}, \frac{1}{2} ; 1 ; x\right)}\right),
$$

where ${ }_{2} F_{1}\left(\frac{1}{2}, \frac{1}{2} ; 1 ; 1-x\right)$ denotes the ordinary or Gaussian hypergeometric function. Then

$$
z:={ }_{2} F_{1}\left(\frac{1}{2}, \frac{1}{2} ; 1 ; x\right)=\varphi^{2}(q),
$$

where $\varphi(q)$ denotes the classical theta function

$$
\varphi(q):=\sum_{n=-\infty}^{\infty} q^{n^{2}} .
$$

The Eisenstein series $Q$ and $R$ can be represented in terms of $z$ and $x$. These representations lead to many further series "evaluations" in terms of $z$ and $x$; see [3, 
pp. 126-139]. Chapter 21 in Ramanujan's second notebook [43], [3, pp. 454-488] is entirely devoted to Eisenstein series. Eisenstein series arise in Ramanujan's "cubic" theory of elliptic functions; see [5, pp. 105-108]. Chapter 15 in his second notebook contains some of the results from [39] and other results not recorded in [39]; see [2, pp. 326-333]. Our brief summary has been by no means exhaustive; for further results of Ramanujan on Eisenstein series, consult [2]-[5].

The present survey of results on Eisenstein series found mostly in Ramanujan's lost notebook [44] comprises entries of different natures. In Section 2, we discuss Ramanujan's formulas for the coefficients of quotients of Eisenstein series. These were communicated to G. H. Hardy from a nursing home in 1918 and greatly extend the content of their last joint paper [24], [42, pp. 310-321]. In Section 3, we examine the role of Eisenstein series in proving congruences for the partition function $p(n)$. This material is found in a manuscript of Ramanujan on the partition and tau functions first published in handwritten form in [44] and then in [12] with commentary. The results in these two sections bring us to the natural investigation of possible congruences for the coefficients of quotients of Eisenstein series upon which we briefly focus in Section 4. At various places in his lost notebook, Ramanujan gives formulas for Eisenstein series in terms of quotients of Dedekind eta-functions. Several examples are given in Section 5. In [39], Ramanujan expresses families of Eisenstein series and related series as polynomials in $P, Q$, and $R$. A page in the lost notebook [44, p. 188] is devoted to another family of series, which in this case is related to the pentagonal number theorem; see Section 6 for some of these series. On another page in the lost notebook [44, p. 211], Ramanujan cryptically relates some formulas for Eisenstein series, which yield approximations to $\pi$ in a spirit not unlike that for approximations to $\pi$ given in his famous paper on modular equations and approximations to $\pi$ [38]. We also indicate in Section 7 how Ramanujan's ideas lead to new series representations for $1 / \pi$. In the final section, we discuss integrals of Eisenstein series associated with Dirichlet characters.

Lastly, we introduce further notation that we use in the sequel. As usual, set

$$
(a ; q)_{\infty}:=\prod_{n=0}^{\infty}\left(1-a q^{n}\right), \quad|q|<1 .
$$

Define, after Ramanujan,

$$
f(-q):=(q ; q)_{\infty}=: e^{-2 \pi i \tau / 24} \eta(\tau), \quad q=e^{2 \pi i \tau}, \quad \operatorname{Im} \tau>0,
$$

where $\eta$ denotes the Dedekind eta-function.

\section{Formulas for the Coefficients of Quotients of Eisenstein Series}

In their famous paper [23], [42, pp. 276-309], Hardy and Ramanujan found an asymptotic formula for the partition function $p(n)$, which arises from the power series coefficients of the reciprocal of the Dedekind eta-function, a modular form of weight $\frac{1}{2}$. As they indicated near the end of their paper, their methods also apply to several analogues of the partition function generated by modular forms of negative weight that are analytic in the upper half-plane. In their last published paper [24], [42, pp. 310 $321]$, they considered a similar problem for the coefficients of modular forms of negative 
weight having one simple pole in a fundamental region, and, in particular, they applied their theorem to find interesting series representations for the coefficients of the reciprocal of the Eisenstein series $E_{6}(\tau)$. Although there are some similarities in the methods of these papers, the principal ideas are quite different in [24] from those in [23]. The ideas in [24] have been greatly extended by H. Poincaré [36], H. Petersson [33], [34], [35], and J. Lehner [27], [28].

While confined to nursing homes and sanitariums during his last two years in England, Ramanujan wrote several letters to Hardy about the coefficients in the power series expansions of certain quotients of Eisenstein series. These letters are photocopied in [44, pp. 97-126] and set to print with commentary in the book by Berndt and Rankin [13, pp. 175-191]. The letters contain many formulas for the coefficients of quotients of Eisenstein series not examined by Hardy and Ramanujan in [24]. Many of Ramanujan's claims do not fall under the purview of the main theorem in [24]. Ramanujan obviously wanted another example to be included in their paper [24], for in his letter of 28 June 1918 [13, pp. 182-183], he wrote, "I am sending you the analogous results in case of $g_{2}$. Please mention them in the paper without proof. After all we have got only two neat examples to offer, viz. $g_{2}$ and $g_{3}$. So please don't omit the results." This letter was evidently written after galley proofs for [24] were printed, because Ramanujan's request went unheeded. The functions $g_{2}$ and $g_{3}$ are the familiar invariants in the theory of elliptic functions and are constant multiples of the Eisenstein series $E_{4}(\tau)$ and $E_{6}(\tau)$, respectively.

Because the example from Hardy and Ramanujan's paper [24] is necessary for us in our examination of the deepest result from Ramanujan's letters, we state it below.

Theorem 2.1. Define the coefficients $p_{n}$ by

$$
\frac{1}{R\left(q^{2}\right)}=: \sum_{n=0}^{\infty} p_{n} q^{2 n}
$$

where, say, $|q|<q_{0}<1$. Let

$$
\mu=2^{a} \prod_{j=1}^{r} p_{j}^{a_{j}},
$$

where $a=0$ or $1, p_{j}$ is a prime of the form $4 m+1$, and $a_{j}$ is a nonnegative integer, $1 \leq j \leq r$. Then, for $n \geq 0$,

$$
p_{n}=\sum_{(\mu)} T_{\mu}(n),
$$

where $\mu$ runs over all integers of the form (2.1), and where

$$
\begin{gathered}
T_{1}(n)=\frac{2}{Q^{2}\left(e^{-2 \pi}\right)} e^{2 n \pi}, \\
T_{2}(n)=\frac{2}{Q^{2}\left(e^{-2 \pi}\right)} \frac{(-1)^{n}}{2^{4}} e^{n \pi},
\end{gathered}
$$


and, for $\mu>2$,

$$
T_{\mu}(n)=\frac{2}{Q^{2}\left(e^{-2 \pi}\right)} \frac{e^{2 n \pi / \mu}}{\mu^{4}} \sum_{c, d} 2 \cos \left((a c+b d) \frac{2 \pi n}{\mu}+8 \tan ^{-1} \frac{c}{d}\right),
$$

where the sum is over all pairs $(c, d)$, where $(c, d)$ is a distinct solution to $\mu=c^{2}+d^{2}$ and $(a, b)$ is any solution to $a d-b c=1$. Also, distinct solutions $(c, d)$ to $\mu=c^{2}+d^{2}$ give rise to distinct terms in the sum in (2.4).

All of Ramanujan's formulas for the coefficients of quotients of Eisenstein series were established in two papers by Berndt and Bialek [6] and by Berndt, Bialek, and Yee [7]. The results proved in the former paper require only a mild extension of Hardy and Ramanujan's principal theorem. However, those in the latter paper are more difficult to prove, and not only did the theorem of Hardy and Ramanujan [24] needed to be extended, but the theorems of Poincaré, Petersson, and Lehner needed to be extended to cover double poles.

As one can see from Theorem 2.1, the formulas for these coefficients have a completely different shape from those arising from modular forms analytic in the upper half-plane. Moreover, these series are very rapidly convergent, more so than those arising from modular forms analytic in the upper half-plane, so that truncating a series, even with a small number of terms, provides a remarkable approximation. This is really very surprising, for when one examines the formulas for these coefficients, one would never guess how such amazingly accurate approximations could be obtained from just two terms.

We now offer an extension of the main theorems of Hardy and Ramanujan [24] and the aforementioned authors. Our theorem can be extended to vastly more general groups and to functions having several simple and double poles, but we only need an application for modular forms with only one double pole on a fundamental region for $\Gamma_{0}(2)$, and so we confine our statement to only this special case.

Theorem 2.2. Suppose that $f(q)=f\left(e^{\pi i \tau}\right)=\phi(\tau)$ is analytic for $q=0$, is meromorphic in the unit circle, and satisfies the functional equation

$$
\phi(\tau)=\phi\left(\frac{a \tau+b}{c \tau+d}\right)(c \tau+d)^{n}
$$

where $a, b, c, d \in \mathbf{Z} ; a d-b c=1 ; c$ is even; and $n \in \mathbf{Z}^{+}$. Assume that $\phi(\tau)$ has only one pole in a fundamental region for $\Gamma_{0}(2)$, a double pole at $\tau=\alpha$. Suppose that $f(q)$ and $\phi(\tau)$ have the Laurent expansions,

$$
\phi(\tau)=\frac{r_{2}}{(\tau-\alpha)^{2}}+\frac{r_{1}}{\tau-\alpha}+\cdots=\frac{\ell_{2}}{\left(q-e^{\pi i \alpha}\right)^{2}}+\frac{\ell_{1}}{q-e^{\pi i \alpha}}+\cdots=f(q) .
$$

Then

$$
\begin{aligned}
f(q)= & 2 \pi i \sum_{c, d}\left\{\frac{c r_{2}(n+2)}{(c \alpha+d)^{n+3}}-\frac{r_{1}}{(c \alpha+d)^{n+2}}\right\} \frac{1}{1-(q / \underline{q})^{2}} \\
& -4 \pi^{2} r_{2} \sum_{c, d} \frac{1}{(c \alpha+d)^{n+4}} \frac{(q / \underline{q})^{2}}{\left(1-(q / \underline{q})^{2}\right)^{2}}, \quad|q|<1,
\end{aligned}
$$


where

$$
\underline{q}=\exp \left(\left(\frac{a \alpha+b}{c \alpha+d}\right) \pi i\right)
$$

and the summation runs over all pairs of coprime integers $(c, d)$ (with c even) which yield distinct values for the set $\{\underline{q},-\underline{q}\}$, and $a$ and $b$ are any integral solutions of

$$
a d-b c=1 \text {. }
$$

Furthermore,

$$
r_{1}=-\frac{i \ell_{1}}{\pi e^{\pi i \alpha}}+\frac{i \ell_{2}}{\pi e^{2 \pi i \alpha}} \quad \text { and } \quad r_{2}=-\frac{\ell_{2}}{\pi^{2} e^{2 \pi i \alpha}}
$$

Now define

$$
B(q):=1+24 \sum_{k=1}^{\infty} \frac{(2 k-1) q^{2 k-1}}{1-q^{2 k-1}}, \quad|q|<1,
$$

and define the coefficients $b_{n}$ by

$$
\frac{1}{B(q)}=\sum_{n=0}^{\infty} b_{n} q^{n}
$$

where $|q|$ is sufficiently small. A formula for $b_{n}$ was claimed by Ramanujan in one of his letters to Hardy and was proved by Berndt, Bialek, and Yee in [7]. We focus in this survey on the coefficients of $1 / B^{2}(q)$, however.

It is not difficult to see that $B(q)$ is the unique modular form of weight 2 with multiplier system identically equal to 1 on the modular group $\Gamma_{0}(2)$. It is also easy to show that $B(q)$ is related to $P(q)$ by the simple formula

$$
B(q)=2 P\left(q^{2}\right)-P(q) .
$$

We now state our principal application of Theorem 2.2.

Theorem 2.3. Define the coefficients $b_{n}^{\prime}$ by

$$
\frac{1}{B^{2}\left(q^{2}\right)}=: \sum_{n=0}^{\infty} b_{n}^{\prime} q^{2 n}
$$

where, say, $|q|<q_{0}<1$. Then,

$$
b_{n}^{\prime}=18 \sum_{\left(\mu_{e}\right)}\left(n+\frac{3 \mu_{e}}{2 \pi}\right) T_{\mu_{e}}(n),
$$

where the sum is over all even integers $\mu_{e}$ of the form (2.1), and where $T_{\mu_{e}}(n)$ is defined by $(2.2)-(2.4)$.

Using Mathematica, we calculated $b_{n}^{\prime}, 1 \leq n \leq 10$, and the first two terms in (2.5). The accuracy is remarkable. 


\begin{tabular}{c|rr}
$n$ & \multicolumn{1}{|c}{} & $18\left(\left(n+\frac{3}{\pi}\right) T_{2}(n)+\left(n+\frac{15}{\pi}\right) T_{10}(n)\right)$ \\
\hline 1 & -48 & -48.001187 \\
2 & 1,680 & $1,679.997897$ \\
3 & $-52,032$ & $-52,031.997988$ \\
4 & $1,508,496$ & $1,508,496.002778$ \\
5 & $-41,952,672$ & $-41,952,671.998915$ \\
6 & $1,133,840,832$ & $1,133,840,831.996875$ \\
7 & $-30,010,418,304$ & $-30,010,418,304.008563$ \\
8 & $781,761,426,576$ & $781,761,426,576.003783$ \\
9 & $-20,110,673,188,848$ & $-20,110,673,188,847.986981$ \\
10 & $512,123,093,263,584$ & $512,123,093,263,584.006307$
\end{tabular}

To further examine the rapidity of convergence of (2.5), we calculated the coefficients of $1 / B^{2}(q)$ up to $n=50$. For $n=20,30,40$, and 50, the coefficients have, respectively, $29,43,57$, and 70 digits, while two-term approximations give, respectively, 29, 42, 55, and 66 of these digits.

\section{Eisenstein Series and Partitions}

Ramanujan utilized Eisenstein series to establish congruences for the partition function $p(n)$. Among his most famous identities connected with partitions are the following four identities:

$$
\begin{aligned}
\sum_{n=1}^{\infty}\left(\frac{n}{5}\right) \frac{q^{n}}{\left(1-q^{n}\right)^{2}} & =q \frac{\left(q^{5} ; q^{5}\right)_{\infty}^{5}}{(q ; q)_{\infty}} \\
1-5 \sum_{n=1}^{\infty}\left(\frac{n}{5}\right) \frac{n q^{n}}{1-q^{n}} & =\frac{(q ; q)_{\infty}^{5}}{\left(q^{5} ; q^{5}\right)_{\infty}} \\
\sum_{n=1}^{\infty}\left(\frac{n}{7}\right) q^{n} \frac{1+q^{n}}{\left(1-q^{n}\right)^{3}} & =q(q ; q)_{\infty}^{3}\left(q^{7} ; q^{7}\right)_{\infty}^{3}+8 q^{2} \frac{\left(q^{7} ; q^{7}\right)_{\infty}^{7}}{(q ; q)_{\infty}}
\end{aligned}
$$

and

$$
8-7 \sum_{n=1}^{\infty}\left(\frac{n}{7}\right) \frac{n^{2} q^{n}}{1-q^{n}}=49 q(q ; q)_{\infty}^{3}\left(q^{7} ; q^{7}\right)_{\infty}^{3}+8 \frac{(q ; q)_{\infty}^{7}}{\left(q^{7} ; q^{7}\right)_{\infty}}
$$

where $|q|<1$ and $\left(\frac{n}{p}\right)$ denotes the Legendre symbol. Identity (3.1) can be utilized to prove Ramanujan's celebrated congruence $p(5 n+4) \equiv 0(\bmod 5)$, while $(3.3)$ can be employed to establish Ramanujan's equally famous congruence $p(7 n+5) \equiv 0(\bmod 7)$. The identities in (3.2) and (3.4) are Eisenstein series on the congruence subgroups $\Gamma_{0}(5)$ and $\Gamma_{0}(7)$, respectively. These four identities were first recorded in an unpublished manuscript of Ramanujan on the partition and tau functions, first published in handwritten form with Ramanujan's lost notebook [44, pp. 135-177]. Equivalent forms of (3.1) and (3.3) are offered by Ramanujan in his first paper on congruences for $p(n)$ [40], [42, pp. 210-213]. Hardy extracted some of the material from this unpublished 
manuscript for Ramanujan's posthumously published paper [41], [42, pp. 232-238]. A typed version of Ramanujan's unpublished manuscript, together with proofs and commentary, has been prepared by Berndt and K. Ono [12]. The latter paper and the new edition of Ramanujan's Collected Papers [42, pp. 372-375] contain several references to proofs of (3.1)-(3.4). H. H. Chan [19] has shown the equivalence of (3.1) and (3.2), as well as the equivalence of (3.3) and (3.4). To the best of our knowledge, (3.4) was first proved by N. J. Fine [22]. See also a paper by O. Kolberg [26] for another proof.

We provide here one example from [12] to illustrate how Ramanujan used Eisenstein series to establish the congruence $p(7 n+5) \equiv 0(\bmod 7), n \geq 0$. Further examples can be found in [12] and [41].

Ramanujan [39, Table I] evidently was the first to observe that

$$
Q^{2}=1+480 \sum_{n=1}^{\infty} \frac{n^{7} q^{n}}{1-q^{n}}
$$

The identity (3.5) also follows readily from the theory of modular forms, since both $E_{4}^{2}$ and $E_{8}$ are modular forms of weight 8 on the full modular group, and the vector space of these forms has dimension 1. It is easy to see from (3.5) and (1.2)-(1.4) that

$$
Q^{2}=P+7 J \quad \text { and } \quad R=1+7 J,
$$

where $J$ (not the same at each occurrence) is a power series in $q$ with integral coefficients. It follows from (3.6) that

$$
\left(1728 q(q ; q)_{\infty}^{24}\right)^{2}=\left(Q^{3}-R^{2}\right)^{2}=P^{3}-2 P Q+R+7 J
$$

Now [39, Tables II and III, resp.]

$$
P Q-R=720 \sum_{n=1}^{\infty} n \sigma_{3}(n) q^{n}
$$

and

$$
P^{3}-3 P Q+2 R=-1728 \sum_{n=1}^{\infty} n^{2} \sigma_{1}(n) q^{n} .
$$

Furthermore, we easily see that

$$
(q ; q)_{\infty}^{48}=\frac{(q ; q)_{\infty}^{49}}{(q ; q)_{\infty}}=\frac{\left(q^{49} ; q^{49}\right)_{\infty}}{(q ; q)_{\infty}}+7 J
$$

It follows from (3.7)-(3.10) that

$$
q^{2} \frac{\left(q^{49} ; q^{49}\right)_{\infty}}{(q ; q)_{\infty}}=\sum_{n=1}^{\infty}\left\{n^{2} \sigma_{1}(n)-n \sigma_{3}(n)\right\} q^{n}+7 J .
$$

In other words,

$$
\left(q^{49} ; q^{49}\right)_{\infty} \sum_{n=0}^{\infty} p(n) q^{n+2}=\sum_{n=1}^{\infty}\left\{n^{2} \sigma_{1}(n)-n \sigma_{3}(n)\right\} q^{n}+7 J
$$


It immediately follows that, for $n \geq 1$,

$$
p(7 n-2) \equiv 0(\bmod 7),
$$

and, as a bonus,

$$
\begin{gathered}
p(n-2)-p(n-51)-p(n-100)+p(n-247) \\
+p(n-345)-\cdots \equiv n^{2} \sigma_{1}(n)-n \sigma_{3}(n)(\bmod 7),
\end{gathered}
$$

where $2,51,100,247, \ldots$ are the numbers of the form $\frac{1}{2}(7 \nu+1)(21 \nu+4)$ and $\frac{1}{2}(7 \nu-1)(21 \nu-4)$.

\section{Congruences for the Coefficients of Quotients of Eisenstein Series}

In calculating the coefficients of the quotients of the Eisenstein series which appear in [6] and [14], and which we briefly discussed in Section 2, we noticed that for some quotients the coefficients in certain arithmetic progressions are divisible by prime powers, usually a power of 3 . In view of Ramanujan's famous congruences for $p(n)$, one of which we proved in the previous section, it seemed natural for us to systematically investigate congruences of this type for Eisenstein series. In some cases, it was very easy to establish our observations, but in other cases, the task was considerably more difficult. We summarize what we have accomplished [15] in the following table. For each quotient, set $F(q)=\sum_{n=0}^{\infty} a_{n} q^{n}$.

\begin{tabular}{|c|rl|ll|}
\hline$F(q)$ & $n \equiv 2$ & $(\bmod 3)$ & $n \equiv 4$ & $(\bmod 8)$ \\
\hline $1 / P(q)$ & $a_{n} \equiv 0$ & $\left(\bmod 3^{4}\right)$ & & \\
\hline $1 / Q(q)$ & $a_{n} \equiv 0$ & $\left(\bmod 3^{2}\right)$ & & \\
\hline $1 / R(q)$ & $a_{n} \equiv 0$ & $\left(\bmod 3^{3}\right)$ & $a_{n} \equiv 0$ & $\left(\bmod 7^{2}\right)$ \\
\hline$P(q) / Q(q)$ & $a_{n} \equiv 0$ & $\left(\bmod 3^{3}\right)$ & & \\
\hline$P(q) / R(q)$ & $a_{n} \equiv 0$ & $\left(\bmod 3^{2}\right)$ & \\
\hline$Q(q) / R(q)$ & $a_{n} \equiv 0$ & $\left(\bmod 3^{3}\right)$ & & \\
\hline$P^{2}(q) / R(q)$ & $a_{n} \equiv 0$ & $\left(\bmod 3^{5}\right)$ & & \\
\hline
\end{tabular}

5. Representations of Eisenstein Series as Quotients of Dedekind ETA-FUNCTIONS

In Section 3, we offered some identities for Eisenstein series that are useful for the study of $p(n)$. The eight identities we present in this section are found on pages 44, 50, 51, and 53 in Ramanujan's lost notebook [44] and were first proved by S. Raghavan and S. S. Rangachari [37]. Since they used the theory of modular forms, it seemed desirable to construct proofs in the spirit of Ramanujan to gain a better insight into how Ramanujan originally discovered them and to also seek a better understanding of the identities themselves. To that end, Berndt, Chan, J. Sohn, and S. H. Son [11] found proofs which depend only on material found in Ramanujan's notebooks [43]. All these identities for Eisenstein series are connected with modular equations of either degree 5 or degree 7. Z.-G. Liu [29] has recently constructed proofs of Ramanujan's septic identities and some new septic identities as well using the theory of elliptic functions, but employing methods from complex analysis. The methods in [11] do not use complex analysis. 
We now record the eight identities in four separate theorems, after which we offer a few words about proofs.

Theorem 5.1 (p. 50). For $Q(q)$ and $f(-q)$ defined by (1.3) and (1.6), respectively,

$$
Q(q)=\frac{f^{10}(-q)}{f^{2}\left(-q^{5}\right)}+250 q f^{4}(-q) f^{4}\left(-q^{5}\right)+3125 q^{2} \frac{f^{10}\left(-q^{5}\right)}{f^{2}(-q)}
$$

and

$$
Q\left(q^{5}\right)=\frac{f^{10}(-q)}{f^{2}\left(-q^{5}\right)}+10 q f^{4}(-q) f^{4}\left(-q^{5}\right)+5 q^{2} \frac{f^{10}\left(-q^{5}\right)}{f^{2}(-q)} .
$$

Theorem 5.2 (p. 51). For $f(-q)$ and $R(q)$ defined by (1.6) and (1.4), respectively,

$$
\begin{aligned}
R(q)= & \left(\frac{f^{15}(-q)}{f^{3}\left(-q^{5}\right)}-500 q f^{9}(-q) f^{3}\left(-q^{5}\right)-15625 q^{2} f^{3}(-q) f^{9}\left(-q^{5}\right)\right) \\
& \times \sqrt{1+22 q \frac{f^{6}\left(-q^{5}\right)}{f^{6}(-q)}+125 q^{2} \frac{f^{12}\left(-q^{5}\right)}{f^{12}(-q)}}
\end{aligned}
$$

and

$$
\begin{aligned}
R\left(q^{5}\right)= & \left(\frac{f^{15}(-q)}{f^{3}\left(-q^{5}\right)}+4 q f^{9}(-q) f^{3}\left(-q^{5}\right)-q^{2} f^{3}(-q) f^{9}\left(-q^{5}\right)\right) \\
& \times \sqrt{1+22 q \frac{f^{6}\left(-q^{5}\right)}{f^{6}(-q)}+125 q^{2} \frac{f^{12}\left(-q^{5}\right)}{f^{12}(-q)}} .
\end{aligned}
$$

Theorem 5.3. For $|q|<1$,

$$
\begin{aligned}
Q(q)= & \left(\frac{f^{7}(-q)}{f\left(-q^{7}\right)}+5 \cdot 7^{2} q f^{3}(-q) f^{3}\left(-q^{7}\right)+7^{4} q^{2} \frac{f^{7}\left(-q^{7}\right)}{f(-q)}\right) \\
& \times\left(\frac{f^{7}(-q)}{f\left(-q^{7}\right)}+13 q f^{3}(-q) f^{3}\left(-q^{7}\right)+49 q^{2} \frac{f^{7}\left(-q^{7}\right)}{f(-q)}\right)^{1 / 3}
\end{aligned}
$$

and

$$
\begin{aligned}
Q\left(q^{7}\right)= & \left(\frac{f^{7}(-q)}{f\left(-q^{7}\right)}+5 q f^{3}(-q) f^{3}\left(-q^{7}\right)+q^{2} \frac{f^{7}\left(-q^{7}\right)}{f(-q)}\right) \\
& \times\left(\frac{f^{7}(-q)}{f\left(-q^{7}\right)}+13 q f^{3}(-q) f^{3}\left(-q^{7}\right)+49 q^{2} \frac{f^{7}\left(-q^{7}\right)}{f(-q)}\right)^{1 / 3}
\end{aligned}
$$

Theorem 5.4. For $|q|<1$,

$$
\begin{aligned}
R(q)= & \left(\frac{f^{7}(-q)}{f\left(-q^{7}\right)}-7^{2}(5+2 \sqrt{7}) q f^{3}(-q) f^{3}\left(-q^{7}\right)-7^{3}(21+8 \sqrt{7}) q^{2} \frac{f^{7}\left(-q^{7}\right)}{f(-q)}\right) \\
& \times\left(\frac{f^{7}(-q)}{f\left(-q^{7}\right)}-7^{2}(5-2 \sqrt{7}) q f^{3}(-q) f^{3}\left(-q^{7}\right)-7^{3}(21-8 \sqrt{7}) q^{2} \frac{f^{7}\left(-q^{7}\right)}{f(-q)}\right)
\end{aligned}
$$


and

$$
\begin{aligned}
R\left(q^{7}\right)= & \left(\frac{f^{7}(-q)}{f\left(-q^{7}\right)}+(7+2 \sqrt{7}) q f^{3}(-q) f^{3}\left(-q^{7}\right)+(21+8 \sqrt{7}) q^{2} \frac{f^{7}\left(-q^{7}\right)}{f(-q)}\right) \\
& \times\left(\frac{f^{7}(-q)}{f\left(-q^{7}\right)}+(7-2 \sqrt{7}) q f^{3}(-q) f^{3}\left(-q^{7}\right)+(21-8 \sqrt{7}) q^{2} \frac{f^{7}\left(-q^{7}\right)}{f(-q)}\right) .
\end{aligned}
$$

Two different methods were used in [11] to prove Theorems 5.1-5.4, but both employ modular equations discovered by Ramanujan and found in his notebooks [43]. The second method seems more amenable to generalization.

Ramaujan recorded several further, related identities in his lost notebook for $P, Q$, and $R$. Some of these involve differential equations satisfied by $P$. See the paper by Berndt, Chan, Sohn, and Son [11] for statements and proofs.

\section{Eisenstein Series and a Series Related to the Pentagonal Number Theorem}

Page 188 of Ramanujan's lost notebook, in the pagination of [44], is devoted to the series

$T_{2 k}:=T_{2 k}(q):=1+\sum_{n=1}^{\infty}(-1)^{n}\left\{(6 n-1)^{2 k} q^{n(3 n-1) / 2}+(6 n+1)^{2 k} q^{n(3 n+1) / 2}\right\}, \quad|q|<1$.

Note that the exponents $n(3 n \pm 1) / 2$ are the generalized pentagonal numbers. Ramanujan recorded formulas for $T_{2 k}, k=1,2, \ldots, 6$, in terms of the Eisenstein series, $P, Q$, and $R$. The first three are given by

$$
\begin{aligned}
& \frac{T_{2}(q)}{(q ; q)_{\infty}}=P, \\
& \frac{T_{4}(q)}{(q ; q)_{\infty}}=3 P^{2}-2 Q, \\
& \frac{T_{6}(q)}{(q ; q)_{\infty}}=15 P^{3}-30 P Q+16 R .
\end{aligned}
$$

Ramanujan's work on this page can be considered as a continuation of his study of representing certain kinds of series as polynomials in Eisenstein series in [39], [42, pp. 136-162].

The first formula, (i), has an interesting arithmetical interpretation. For $n \geq 1$, let $\sigma(n)=\sum_{d \mid n} d$, and define $\sigma(0)=-\frac{1}{24}$. Let $n$ denote a nonnegative integer. Then

$$
-24 \sum_{j+k(3 k \pm 1) / 2=n}(-1)^{k} \sigma(j)= \begin{cases}(-1)^{r}(6 r-1)^{2}, & \text { if } n=r(3 r-1) / 2 \\ (-1)^{r}(6 r+1)^{2}, & \text { if } n=r(3 r+1) / 2 \\ 0, & \text { otherwise }\end{cases}
$$

where the sum is over all nonnegative pairs of integers $(j, k)$ such that $j+k(3 k \pm 1) / 2=$ $n$.

There are many identities involving the divisor sums $\sigma_{k}(n):=\sum_{d \mid n} d^{k}$ in the literature, but we have not previously seen (6.1). Besides (6.1), other identities of Ramanujan 
can be reformulated in terms of divisor sums. In particular, see [2, pp. 326-329] and the references cited there. The most thorough study of identities of this sort has been undertaken by J. G. Huard, Z. M. Ou, B. K. Spearman, and K. S. Williams [25], where many references to the literature can also be found.

In general, $T_{2 k}(q)$ can be represented as a polynomial in terms of the form $P^{2 a} Q^{4 b} R^{6 c}$, where $2 a+4 b+6 c=2 k$. It seems to be extremely difficult to find a general formula for this polynomial, but on page 188, Ramanujan does give the "first" five terms. Define the polynomials $f_{2 k}(P, Q, R), k \geq 1$, by

Then, for $k \geq 1$,

$$
f_{2 k}(P, Q, R):=\frac{T_{2 k}(q)}{(q ; q)_{\infty}}
$$

$$
\begin{aligned}
f_{2 k}(P, Q, R)= & \cdot 3 \cdots(2 k-1)\left\{P^{k}-\frac{k(k-1)}{3} P^{k-2} Q+\frac{8 k(k-1)(k-2)}{45} P^{k-3} R\right. \\
& -\frac{11 k(k-1)(k-2)(k-3)}{210} P^{k-4} Q^{2} \\
& \left.+\frac{152 k(k-1)(k-2)(k-3)(k-4)}{14175} P^{k-5} Q R+\cdots\right\} .
\end{aligned}
$$

Proofs of all the claims in this section can be found in a paper by Berndt and Yee [14].

\section{Eisenstein Series and Approximations to $\pi$}

On page 211 in his lost notebook, in the pagination of [44], Ramanujan listed eight integers, $11,19,27,43,67,163,35$, and 51 at the left margin. To the right of each integer, Ramanujan recorded a linear equation in $Q^{3}$ and $R^{2}$. The arguments $q$ in these identities were not revealed by Ramanujan, but $q=-\exp (-\pi \sqrt{n})$, where $n$ is the integer at the left margin. To the right of each equation in $Q^{3}$ and $R^{2}$, Ramanujan entered an equality involving $\pi$ and square roots. (For the integer 51, the linear equation and the equality involving $\pi$, in fact, are not recorded by Ramanujan.) The equalities in the third column lead to approximations of $\pi$ that are remindful of approximations given by Ramanujan in his famous paper on modular equations and approximations to $\pi$ [38], [42, p. 33].

We offer a general theorem from which all the equalities in the third column follow. Considerable notation is first needed, however.

Let

$$
P_{n}:=P\left(-e^{-\pi \sqrt{n}}\right), \quad Q_{n}:=Q\left(-e^{-\pi \sqrt{n}}\right), \quad \text { and } \quad R_{n}:=R\left(-e^{-\pi \sqrt{n}}\right) .
$$

Recall that the modular $j$-invariant $j(\tau)$ is defined by

$$
j(\tau)=1728 \frac{Q^{3}(q)}{Q^{3}(q)-R^{2}(q)}, \quad q=e^{2 \pi i \tau}, \quad \operatorname{Im} \tau>0 .
$$

In particular, if $n$ is a positive integer,

$$
j_{n}:=j\left(\frac{3+\sqrt{-n}}{2}\right)=1728 \frac{Q_{n}^{3}}{Q_{n}^{3}-R_{n}^{2}},
$$


where $Q_{n}$ and $R_{n}$ are defined by (7.1). Furthermore, set

$$
J_{n}=-\frac{1}{32} \sqrt[3]{j_{n}}
$$

Next, define

$$
b_{n}=\left\{n\left(1728-j_{n}\right)\right\}^{1 / 2}
$$

and

$$
a_{n}=\frac{1}{6} b_{n}\left\{1-\frac{Q_{n}}{R_{n}}\left(P_{n}-\frac{6}{\pi \sqrt{n}}\right)\right\} .
$$

The numbers $a_{n}$ and $b_{n}$ arise in series representations for $1 / \pi$ proved by D. V. and G. V. Chudnovsky [21] and J. M. and P. B. Borwein [17].

We now have sufficient notation to state our first theorem.

Theorem 7.1. If $P_{n}, b_{n}, a_{n}$, and $J_{n}$ are defined by (7.1), (7.4), (7.5), and (7.3), respectively, then

$$
\frac{1}{\sqrt{Q_{n}}}\left(\sqrt{n} P_{n}-\frac{6}{\pi}\right)=\sqrt{n}\left(1-6 \frac{a_{n}}{b_{n}}\right)\left(\frac{\left(\frac{8}{3} J_{n}\right)^{3}+1}{\left(\frac{8}{3} J_{n}\right)^{3}}\right)^{1 / 2} .
$$

To illustrate Theorem 7.1, we offer Ramanujan's eight examples.

Corollary 7.2. We have

$$
\begin{aligned}
\frac{1}{\sqrt{Q_{11}}}\left(\sqrt{11} P_{11}-\frac{6}{\pi}\right) & =\sqrt{2} \\
\frac{1}{\sqrt{Q_{19}}}\left(\sqrt{19} P_{19}-\frac{6}{\pi}\right) & =\sqrt{6} \\
\frac{1}{\sqrt{Q_{27}}}\left(\sqrt{27} P_{27}-\frac{6}{\pi}\right) & =3 \sqrt{\frac{6}{5}} \\
\frac{1}{\sqrt{Q_{43}}}\left(\sqrt{43} P_{43}-\frac{6}{\pi}\right) & =6 \sqrt{\frac{3}{5}} \\
\frac{1}{\sqrt{Q_{67}}}\left(\sqrt{67} P_{67}-\frac{6}{\pi}\right) & =19 \sqrt{\frac{6}{55}}, \\
\frac{1}{\sqrt{Q_{163}}}\left(\sqrt{163} P_{163}-\frac{6}{\pi}\right) & =362 \sqrt{\frac{3}{3335}} \\
\frac{1}{\sqrt{Q_{35}}}\left(\sqrt{35} P_{35}-\frac{6}{\pi}\right) & =(2+\sqrt{5}) \sqrt{\frac{2}{\sqrt{5}}} \\
\frac{1}{\sqrt{Q_{51}}}\left(\sqrt{51} P_{51}-\frac{6}{\pi}\right) & =.
\end{aligned}
$$

Theorem 7.1 leads to the following theorem giving approximations of $\pi$.

Theorem 7.3. We have

$$
\pi \approx \frac{6}{\sqrt{n}-r_{n}}=: A_{n}
$$


with the error approximately equal to

$$
144 \frac{\sqrt{n}+5 r_{n}}{\left(\sqrt{n}-r_{n}\right)^{2}} e^{-\pi \sqrt{n}}
$$

where $r_{n}$ is the algebraic expression on the right side in (7.6), i.e.,

$$
r_{n}=\sqrt{n}\left(\frac{\left(\frac{8}{3} J_{n}\right)^{3}+1}{\left(\frac{8}{3} J_{n}\right)^{3}}\right)^{1 / 2} .
$$

Theorem 7.3 with the examples above lead to approximations of $\pi$ which we offer in the next table. Let $N_{n}$ denote the number of digits of $\pi$ which agree with the decimal expansion of $A_{n}$.

\begin{tabular}{|l|c|c|}
\hline$n$ & $A_{n}$ & $N_{n}$ \\
\hline 11 & $3.1538 \ldots$ & 1 \\
\hline 19 & $3.1423 \ldots$ & 2 \\
\hline 27 & $3.1416621 \ldots$ & 3 \\
\hline 43 & $3.141593 \ldots$ & 5 \\
\hline 67 & $3.14159266 \ldots$ & 7 \\
\hline 163 & $3.14159265358980 \ldots$ & 12 \\
\hline 35 & $3.141601 \ldots$ & 3 \\
\hline 51 & $3.14159289 \ldots$ & 6 \\
\hline
\end{tabular}

Although not mentioned by Ramanujan on page 211 in [44], the ideas needed to prove the results on this page lead to a very general infinite series expansion for $1 / \pi$ in the spirit of those given by Ramanujan [38], and later by the Chudnovskys [21] and Borweins [17]. To state this expansion, we need further definitions.

Let

$$
\mathbf{J}(q):=\frac{1728}{j\left(\frac{3+\tau}{2}\right)}
$$

and

$$
\mathbf{J}_{n}=\mathbf{J}\left(e^{-\pi \sqrt{n}}\right), \quad n>0 .
$$

Furthermore, let

$$
t_{n}=\frac{Q_{n}}{R_{n}}\left(P_{n}-\frac{6}{\sqrt{n} \pi}\right)
$$

where $P_{n}, Q_{n}$, and $R_{n}$ are defined by (7.1).

We now state a general series representation for $1 / \pi$.

Theorem 7.4. If $\mathbf{J}_{n}$ and $t_{n}$ are defined by (7.7) and (7.8), respectively, then

$$
\frac{6}{\sqrt{n} \sqrt{1-\mathbf{J}_{n}}} \frac{1}{\pi}=\sum_{k=0}^{\infty} \frac{\left(\frac{1}{6}\right)_{k}\left(\frac{5}{6}\right)_{k}\left(\frac{1}{2}\right)_{k}}{(k !)^{3}} \mathbf{J}_{n}^{k}\left(6 k+1-t_{n}\right),
$$

where $(a)_{0}:=1$ and $(a)_{n}:=a(a+1)(a+2) \cdots(a+n-1)$, for each positive integer $n$. 
If $j_{n}$ is defined by $(7.2)$, then

$$
j_{3 n}=-27 \frac{\left(\lambda_{n}^{2}-1\right)\left(9 \lambda_{n}^{2}-1\right)^{3}}{\lambda_{n}^{2}}
$$

where

$$
\lambda_{n}=\frac{e^{\pi \sqrt{n} / 2}}{3 \sqrt{3}} \frac{f^{6}\left(e^{-\pi \sqrt{n / 3}}\right)}{f^{6}\left(e^{-\pi \sqrt{3 n}}\right)}
$$

and $f$ is defined by (1.6). The numbers $\lambda_{n}$ were first defined by Ramanujan on page 212 of his lost notebook, in the pagination of [44]. He recorded an extenssive table of values which were verified by Berndt, Chan, S.-Y. Kang, and L.-C. Zhang [10] by a variety of methods.

In [8], Berndt and Chan used Theorem 7.4 to achieve a new "World Record" for the number of digits of $1 / \pi$ per term. They used the value,

$$
\lambda_{1105}=\left(\frac{\sqrt{5}+1}{2}\right)^{12}(4+\sqrt{17})^{3}\left(\frac{15+\sqrt{221}}{2}\right)^{3}(8+\sqrt{65})^{3},
$$

in the formula (7.10) to calculate $\mathbf{J}_{3315}$ (defined by (7.7)). Substituting this value for $\mathbf{J}_{3315}$ and the value of $t_{3315}$ (defined by (7.8)) in (7.9), Berndt and Chan obtained a series for $1 / \pi$ which yields about 73 or 74 digits of $\pi$ per term. All the results in this section were established in a paper by Berndt and Chan [8].

\section{Integrals of Eisenstein Series}

In the Introduction, we gave one beautiful example of an integral of an Eisenstein series found in the lost notebook. Here we present a different kind of example. At first glance, it does not appear that there is any connection with Eisenstein series. Recall that the functions $f(-q)$ and $\eta(\tau)$ are defined in (1.6).

Theorem 8.1. Suppose that $0<q<1$. Then

$$
q^{1 / 9} \prod_{n=1}^{\infty}\left(1-q^{n}\right)^{\chi(n) n}=\exp \left(-C_{3}-\frac{1}{9} \int_{q}^{1} \frac{f^{9}(-t)}{f^{3}\left(-t^{3}\right)} \frac{d t}{t}\right),
$$

where

$$
C_{3}=\frac{3 \sqrt{3}}{4 \pi} L(2, \chi)=L^{\prime}(-1, \chi) .
$$

Here, $L(s, \chi)$ denotes the Dirichlet L-function defined for Re $s>1$ by $L(s, \chi)=$ $\sum_{n=1}^{\infty} \chi(n) / n^{s}$. Furthermore, in (8.1) and (8.2), $\chi(n)=\left(\frac{n}{3}\right)$, the Legendre symbol.

This result, found on page 207 of Ramanujan's lost notebook, was first proved by Son [46], except that he did not establish Ramanujan's formula (8.2) for $C_{3}$. Berndt and Zaharescu [16] found another proof of Theorem 8.1, which included a proof of (8.2). 
We now explain the connection of Theorem 8.1 with Eisenstein series. A key to the proof of (8.1) is the identity

$$
\frac{f^{9}(-q)}{f^{3}\left(-q^{3}\right)}=1-9 \sum_{n=1}^{\infty} \sum_{d \mid n}\left(\frac{d}{3}\right) d^{2} q^{n}
$$

which was first proved by L. Carlitz [18] and then by Son [46]. The series on the right side of (8.3) is an example of an Eisenstein series $E_{k, \chi}(\tau)$ of weight $k$, defined on the congruence subgroup $\Gamma_{0}(N)$ by

$$
E_{k, \chi}(\tau):=1-\frac{2 k}{B_{k, \chi}} \sum_{n=1}^{\infty} \sum_{d \mid n} \chi(d) d^{k-1} q^{n}, \quad q=e^{2 \pi i \tau},
$$

where $\chi$ is a Dirichlet character of modulus $N$, and $B_{k, \chi}$ denotes the $k$ th generalized Bernoulli number associated with $\chi$.

Ramanujan's identity (3.4), when written in the form,

$$
E_{3, \chi}(z)=1-\frac{7}{8} \sum_{n=1}^{\infty} \sum_{d \mid n} \chi(d) d^{2} q^{n}=\frac{\eta^{7}(\tau)}{\eta(7 \tau)}+\frac{49}{8} \eta^{3}(\tau) \eta^{3}(7 \tau)
$$

provides another example, where now $\chi(n)$ denotes the Legendre symbol modulo 7 . Then (8.5) leads to the identity

$$
q^{8 / 7} \prod_{n=1}^{\infty}\left(1-q^{n}\right)^{\chi(n) n}=\exp \left(-C_{7}-\frac{8}{7} \int_{q}^{1}\left\{\frac{f^{7}(-t)}{f\left(-t^{7}\right)}+\frac{49}{8} t f^{3}(-t) f^{3}\left(-t^{7}\right)\right\} \frac{d t}{t}\right),
$$

where

$$
C_{7}=L^{\prime}(-1, \chi)
$$

Clearly (8.6)and (8.7) are analogues of (8.1) and (8.2), respectively.

S. Ahlgren, Berndt, Yee, and Zaharescu [1] have proved a general theorem from which (8.1) and (8.6) follow as special cases. The integral appearing in these authors' general theorem contains the Eisenstein series (8.4). It is only in certain instances that we have identities of the type (8.3) and (8.5), which enable us to reformulate the identities in terms of integrals of eta-functions, as we have in (8.1) and (8.6).

\section{REFERENCES}

[1] S. Ahlgren, B. C. Berndt, A. J. Yee, and A. Zaharescu, Integrals of Eisenstein series, derivatives of L-functions, and the Dedekind eta-function, submitted for publication.

[2] B. C. Berndt, Ramanujan's Notebooks, Part II, Springer-Verlag, New York, 1989.

[3] B. C. Berndt, Ramanujan's Notebooks, Part III, Springer-Verlag, New York, 1991.

[4] B. C. Berndt, Ramanujan's Notebooks, Part IV, Springer-Verlag, New York, 1994.

[5] B. C. Berndt, Ramanujan's Notebooks, Part V, Springer-Verlag, New York, 1998.

[6] B. C. Berndt and P. R. Bialek, On the power series coefficients of certain quotients of Eisenstein series, submitted for publication.

[7] B. C. Berndt, P. Bialek, and A. J. Yee, Formulas of Ramanujan for the power series coefficients of certain quotients of Eisenstein series, International Mathematics Research Notices, to appear.

[8] B. C. Berndt and H. H. Chan, Eisenstein series and approximations to $\pi$, Illinois J. Math. 45 (2001), 75-90. 
[9] B. C. Berndt, H. H. Chan, and S.-S. Huang, Incomplete elliptic integrals in Ramanujan's lost notebook, in q-series from a Contemporary Perspective, M. E. H. Ismail and D. Stanton, eds., American Mathematical Society, Providence, RI, 2000, pp. 79-126.

[10] B. C. Berndt, H. H. Chan, S.-Y. Kang, and L.-C. Zhang, A certain quotient of eta-functions found in Ramanujan's lost notebook, Pacific J. Math., to appear.

[11] B. C. Berndt, H. H. Chan, J. Sohn, and S. H. Son, Eisenstein series in Ramanujan's lost notebook, Ramanujan J. 4 (2000), 81-114.

[12] B. C. Berndt and K. Ono, Ramanujan's unpublished manuscript on the partition and tau functions with proofs and commentary, Sém. Lotharingien de Combinatoire 42 (1999), 63 pp; The Andrews Festschrift, D. Foata and G.-N. Han, eds., Springer-Verlag, Berlin, 2001, pp. 39-110.

[13] B. C. Berndt and R. A. Rankin, Ramanujan: Letters and Commentary, American Mathematical Society, Providence, RI, 1995; London Mathematical Society, London, 1995.

[14] B. C. Berndt and A. J. Yee, A page on Eisenstein series in Ramanujan's lost notebook, submitted for publication.

[15] B. C. Berndt and A. J. Yee, Congruences for the coefficients of quotients of Eisenstein series, submitted for publication.

[16] B. C. Berndt and A. Zaharescu, An integral of Dedekind eta-functions in Ramanujan's lost notebook, J. Reine Angew. Math., to appear.

[17] J. M. Borwein and P. M. Borwein, More Ramanujan-type series for $1 / \pi$, in Ramanujan Revisited, G. E. Andrews, R. A. Askey, B. C. Berndt, K. G. Ramanathan, and R. A. Rankin, eds., Academic Press, Boston, 1988, pp. 359-374

[18] L. Carlitz, Note on some partition formulae, Quart. J. Math. (Oxford) (2) 4 (1953), 168-172.

[19] H. H. Chan, On the equivalence of Ramanujan's partition identities and a connection with the Rogers-Ramanujan continued fraction, J. Math. Anal. Applics. 198 (1996), 111-120.

[20] H. H. Chan, Representations of integers as sums of 32 squares, submitted for publication.

[21] D. V. Chudnovsky and G. V. Chudnovsky, Approximation and complex multiplication according to Ramanujan, in Ramanujan Revisited, G. E. Andrews, R. A. Askey, B. C. Berndt, K. G. Ramanathan, and R. A. Rankin, eds., Academic Press, Boston, 1988, pp. 375-472.

[22] N. J. Fine, On a system of modular functions connected with the Ramanujan identities, Tohoku Math. J. (2) 8 (1956), 149-164.

[23] G. H. Hardy and S. Ramanujan, Asymptotic formulae in combinatory analysis, Proc. London Math. Soc. (2) 17 (1918), 75-118.

[24] G. H. Hardy and S. Ramanujan, On the coefficients in the expansions of certain modular functions, Proc. Royal Soc. A 95 (1918), 144-155.

[25] J. G. Huard, Z. M. Ou, B. K. Spearman, and K. S. Williams, Elementary evaluation of certain convolution sums involving divisor functions, in Number Theory for the Millennium, Vol. 2, M. A. Bennett, B. C. Berndt, N. Boston, H. G. Diamond, A. J. Hildebrand, and W. Philipp, eds., A K Peters, Natick, MA, to appear.

[26] O. Kolberg, Note on the Eisenstein series of $\Gamma_{0}(p)$, Arb. Univ. Bergen, Mat.-Naturv. Ser., No. 6 (1968), 20 pp.

[27] J. Lehner, The Fourier coefficients of automorphic forms on horocyclic groups, II, Mich. Math. J. 6 (1959), 173-193.

[28] J. Lehner, The Fourier coefficients of automorphic forms on horocyclic groups, III, Mich. Math. J. 7 (1960), 65-74.

[29] Z.-G. Liu, Some Eisenstein series identities related to modular equations of the seventh order, Pacific J. Math., to appear.

[30] Z.-G. Liu, On the reprsentation of integers as sums of squares, in q-Series with Applications to Combinatorics, Number Theory, and Physics, B. C. Berndt and K. Ono, eds., Contemp. Math. No., American Mathematical Society, Providence, RI, 2001, pp. 163-176.

[31] S. Milne, Infinite families of exact sums of squares formulas, Jacobi elliptic functions, continued fractions, and Schur functions, Ramanujan J. 6 (2002), to appear.

[32] K. Ono, Representations of integers as sums of squares, J. Number Thy., to appear. 
[33] H. Petersson, Konstruktion der Modulformen und der zu gewissen Grenzkreisgruppen gehörigen automorphen Formen von positiver reeller Dimension und die vollständige Bestimmung ihrer Fourierkoeffzienten, S.-B. Heidelberger Akad. Wiss. Math. Nat. Kl. (1950), 415-474.

[34] H. Petersson, Über automorphe Orthogonalfunktionen und die Konstruktion der automorphen Formen von positiver reeller Dimension, Math. Ann. 127 (1954), 33-81.

[35] H. Petersson, Über automorphe Formen mit Singularitäten im Diskontinuitätsgebiet, Math. Ann. 129 (1955), 370-390.

[36] H. Poincaré, Oeuvres, Vol. 2, Gauthiers-Villars, Paris, 1916.

[37] S. Raghavan and S. S. Rangachari, On Ramanujan's elliptic integrals and modular identities, in Number Theory and Related Topics, Oxford University Press, Bombay, 1989, pp. 119-149.

[38] S. Ramanujan, Modular equations and approximations to $\pi$, Quart. J. Math. 45 (1914), 350-372.

[39] S. Ramanujan, On certain arithmetical functions, Trans. Cambridge Philos. Soc. 22 (1916), 159184.

[40] S. Ramanujan, Some properties of $p(n)$, the number of partitions of $n$ Proc. Cambridge Philos. Soc. 19 (1919), 207-210.

[41] S. Ramanujan, Congruence properties of partitions, Math. Z. 9 (1921), 147-153.

[42] S. Ramanujan, Collected Papers, Cambridge University Press, Cambridge, 1927; reprinted by Chelsea, New York, 1962; reprinted by the American Mathematical Society, Providence, RI, 2000.

[43] S. Ramanujan, Notebooks (2 volumes), Tata Institute of Fundamental Research, Bombay, 1957.

[44] S. Ramanujan, The Lost Notebook and Other Unpublished Papers, Narosa, New Delhi, 1988.

[45] R. A. Rankin, Modular Forms and Functions, Cambridge University Press, Cambridge, 1977.

[46] S. H. Son, Some integrals of theta functions in Ramanujan's lost notebook, in Fifth Conference of the Canadian Number Theory Association, R. Gupta and K. S. Williams, eds., CRM Proc. and Lecture Notes, Vol. 19, American Mathematical Society, Providence, RI, 1999, pp. 323-332.

Department of Mathematics, University of Illinois, 1409 West Green Street, UrBANA, IL 61801, USA

E-mail address: berndt@math.uiuc.edu

Department of Mathematics, University of Illinois, 1409 West Green Street, URBANA, IL 61801, USA

E-mail address: yee@math.uiuc.edu 